\title{
Evolution of dimorphic traits: effect of directional selection on heritability
}

\author{
DEREK A. ROFF \\ Department of Biology, McGill University, 1205 Dr Penfield Avenue, Montreal, Quebec, Canada H3A $1 B 1$
}

\begin{abstract}
Directional selection on a quantitative character is predicted to decrease genetic variance and lead to rapid fixation of alleles. However, many traits vary in a dichotomous manner although the underlying genetic determination is polygenic. This paper presents a theoretical examination of the effects of directional selection on a dimorphic trait. Such selection can change the incidence of the selected morph from 50 per cent to 99 per cent within 20 generations. However, after an initial decline heritability is predicted to rise back to its original value. The consequences of a finite number of alleles and loci on this prediction are examined using a simulation model. These simulations show that, unlike the case of directional selection on a continuously distributed trait, there is little loss of genetic variance even when the trait is controlled by only three loci with two alleles per locus.
\end{abstract}

Keywords: dimorphism, genetic variance, heritability, selection, threshold traits.

\section{Introduction}

It has long been known that directional selection on a quantitative trait will lead to a decrease in genetic variance and, further, that if the number of loci and alleles is finite, rapid exhaustion of the variance because of fixation (Bulmer, 1976, 1985). The assumption underlying this analysis is that the continuous nature of the trait is phenotypically evident. But many traits occur as discrete, rather than continuously distributed, characters: for example, dimorphic variation in morphological structures in cladocera (Dodson, 1989), beetles and earwigs (Eberhard \& Gutiérrez, 1991); thrips (Crespi, 1988), Hymenoptera (Danforth, 1991; Elmes, 1991); paedomorphosis in amphibia (Harris et al., 1990); wing dimorphism in insects (Roff, 1986a); mating behaviour (see Table 7.7 in Roff, 1992); sex ratio (Bull et al., 1982) and diapause (Mousseau \& Roff, 1989). Although these traits appear phenotypically discrete, their inheritance may be polygenic, the particular manifestation of the trait being a function of a threshold of sensitivity.

The basic genetical model for the analysis of threshold traits is well understood (Falconer, 1989). According to this model a continuously varying character underlies the expression of the trait; individuals with values lying above the threshold will be of one type whereas individuals lying below the threshold will be of the other. Given this model the heritability of the trait can be estimated (Bull et al., 1982; Roff, 1986b, 1990; Falconer, 1989). Heritabilities of ecologically important dimorphic traits vary from moderate to high (Table 1). Continued selection for one particular morph, such as the winged form in a wing dimorphic insect, will shift the frequency of the morph towards monomorphism (Roff, 1990). However, because selection acts on the discrete manifestation of the trait, it is not evident that the previously published analyses of the effects of directional selection on genetic variance are valid. In this paper a theoretical exploration of the effects of directional selection on the genetic variance of a threshold trait is presented.

\section{Theory}

Selection on a threshold trait is a type of truncation selection in that only individuals beyond a certain critical value (threshold) are selected but in this case selection intensity decreases each generation. Suppose the population originally comprises 50 per cent of each morph. In the first generation of selection 50 per cent of the population will be selected. But the second generation consists of greater than 50 per cent of the selected morph and hence the selection intensity on the second generation must be less than on the first. As the proportion of the selected morph increases in frequency the intensity of selection declines. 
Table 1 Heritability estimates for some dimorphic traits in non-domestic populations

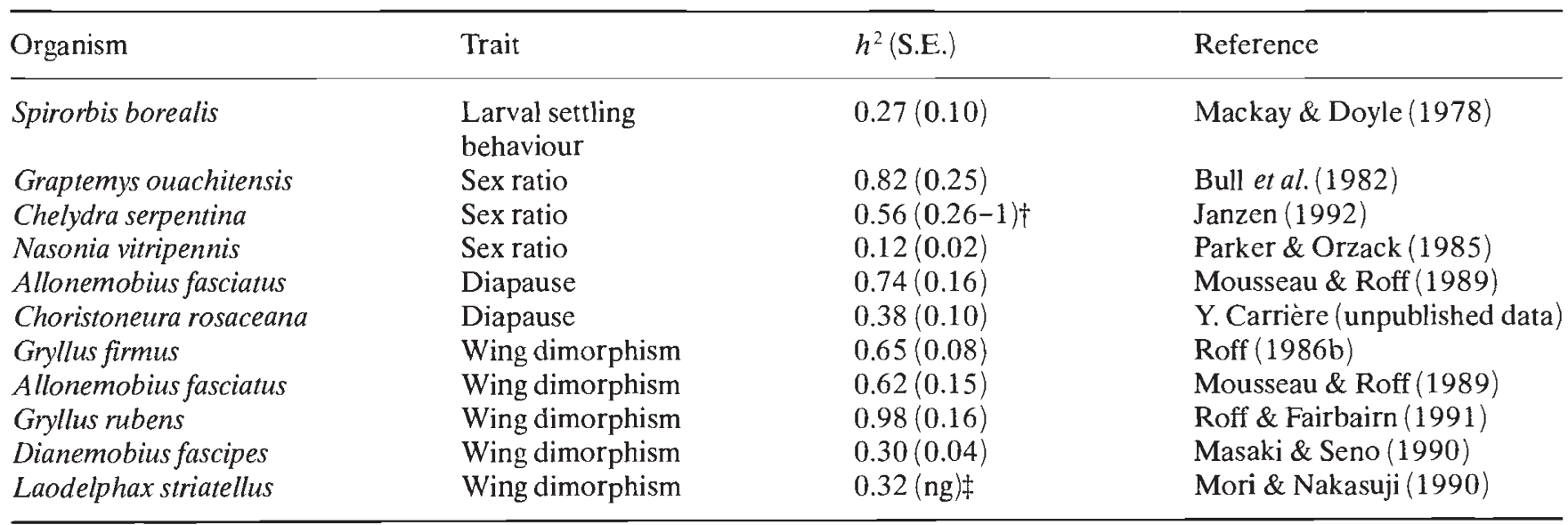

$\dagger$ Asymmetric 95 per cent confidence limits calculated using $\chi^{2}$. łS.E. not given.

The change in genetic variance under truncation selection in which a constant fraction, $p$, of the population is selected each generation is given by (Bulmer, 1985)

$V_{\mathrm{A}}(t)=\frac{1}{2}\left(1-h^{2}(t) i(i-z)\right) V_{\mathrm{A}}(t)+\frac{1}{2} V_{\mathrm{A}}(0)$,

where $V_{\mathrm{A}}(t)$ is the additive genetic variance after $t$ generations of selection, $h^{2}(t)$ is the heritability, $z$ is the standard normal deviate corresponding to the proportion selected, $p$, and $i$ is the intensity of selection. The additive genetic variance, and hence heritability, decreases rapidly during the first few generations of truncation selection but quickly reaches an equilibrium value (Table 2). If selection is relaxed the variance returns to its original value. Selection on a threshold trait produces the same initial decline in genetic variance as for truncation selection but as selection intensity declines as the proportion of the selected phenotype approaches 1 , genetic variance increases and heritability returns to its initial value (Fig. 1).

A central assumption of the above analyses is that of an infinite number of loci or alleles. This assumption is critical, as illustrated by the simulation results of Bulmer (1976) for truncation selection on a quantitative trait. In his model Bulmer assumed 12 unlinked loci, each with two alleles contributing either 1 or 0 to the phenotypic value. Whereas, in accord with theory, the variance decreased in the first five generations of truncation selection, it did not stabilize thereafter and by generation 13 the loci had become fixed. Thus truncation selection with a modest number of loci may quickly lead to the exhaustion of the genetic variance. However, as the selection intensity on a threshold trait necessarily declines during selection and the population approaches random mating, the probability of fixation will be greatly reduced.
Table 2 Predicted changes in heritability under four levels of truncation selection, given an initial heritability of 0.65 and $V_{\mathrm{A}}(0)=100$

\begin{tabular}{lllll}
\hline & \multicolumn{4}{c}{ Proportion selected each generation } \\
\cline { 2 - 5 } Generation & 0.05 & 0.10 & 0.20 & 0.40 \\
\hline 1 & 0.65 & 0.65 & 0.65 & 0.65 \\
2 & 0.57 & 0.58 & 0.58 & 0.59 \\
3 & 0.56 & 0.56 & 0.57 & 0.58 \\
4 & 0.56 & 0.56 & 0.56 & 0.57 \\
5 & 0.56 & 0.56 & 0.56 & 0.57 \\
\hline
\end{tabular}

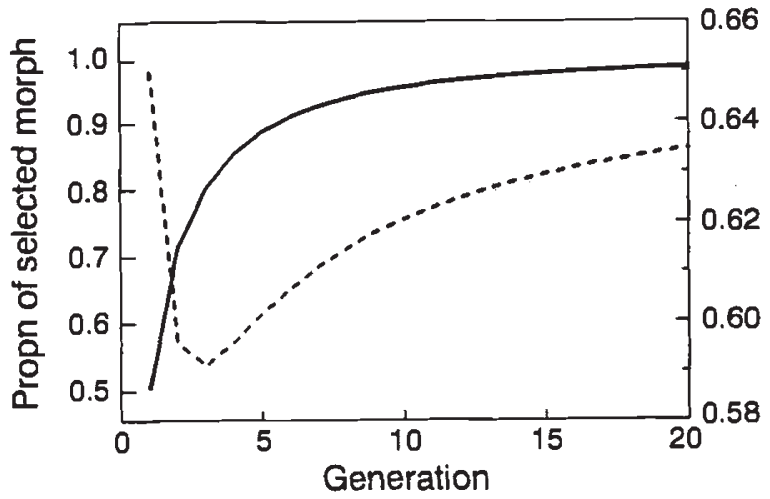

Fig. 1 Prediction from equation 1 of the effect of selection on the proportion of the selected morph (- - , and heritability (- - ) for a dimorphic trait.

To examine the consequences of a finite number of loci and alleles on the change in genetic variance of a threshold trait a simulation model was utilized similar to that of Bulmer (1976), described above. The underlying continuously distributed character was assumed 
to be determined by $n$ unlinked loci each with two alleles contributing either 0 or 1 to the phenotypic value. No dominance or epistasis was assumed and the initial frequency at each locus was set at 50 per cent. The additive genetic variance, assuming Hardy-Weinberg equilibrium and linkage equilibrium in the unselected population, was thus equal to $0.5 n$. The phenotypic value of an individual was obtained as the summed contribution of all loci plus a random normal deviate distributed with zero mean and variance $V_{\mathrm{E}}$. The environmental variance, $V_{E}$, was determined from the heritability in the unselected population (set at 0.65 to match the value obtained for $G$. firmus, see Table 1) and the relationship $h^{2}=V_{\mathrm{A}} /\left(V_{\mathrm{A}}+V_{\mathrm{E}}\right)$. In the founding population both morphs were set at equal frequency, obtained by setting the threshold value equal to $n$. Each generation consisted of 100 families with five offspring per family. All individuals with phenotypic values greater than the threshold value were selected. One hundred pairs were chosen with replacement from the selected population to form the parents of the next generation. In the initial population each individual was heterozygous at all loci: prior to the selection the population was passed through two generations of random mating to achieve Hardy-Weinberg equilibrium. The number of loci was varied from three to 20 , with 20 replicates per $n$.

The mean trajectory of the proportion of the selected morph in the population was remarkably insensitive to the number of loci determining the trait (Fig. 2). For all loci, heritability in the first generation of selection decreased to about 0.4 but thereafter the response depended on the number of loci (Fig. 2). With three loci there was a continuous decline in the genetic variance and by generation 20 the heritability had decreased to about 0.3. However, for the first 19 generations no loci were fixed; in the 20th generation one locus fixed in two of the 20 replicates. A similar pattern was found with four loci, the heritability dropping to 0.4 by generation 20 , and one locus becoming fixed in the 18th generation of one replicate. Fixation of one locus in one replicate line also occurred with five loci but in all other replicates all loci were still heterozygous at generation 20 . With five or more loci the heritability initially dropped but then began to increase, exceeding 0.5 by generation 20 for $n$ equal to 10,15 and 20. Variation in individual lines was quite considerable and in any experiment a decline in genetic variance may not be evident (Fig. 3).

The fixation of loci is due to both genetic drift and directional selection. The relative importance of these two effects for the case of 10 loci is illustrated in Figs 4 and 5. Figure 4 shows the results from simulations in which the model was run for 200 generations under three regimes: firstly, no selection applied, secondly, selection discontinued at generation 50 and thirdly, selection continued throughout. Population size was fixed at 500 and each regime was replicated fivefold. In the absence of selection, drift alone leads to the fixation of 1.8 loci, on average, whereas continuous selection fixes 6.2 loci after 200 generations (Fig. 4). As shown by the second regimen (no selection after generation $50)$, the fixation of the additional 4.4 loci is not entirely attributable to selection. Selection for 50 generations fixes 0.2 loci but shifts the allele frequencies away from 50 per cent. As a consequence, even when selection is discontinued there is an increased probability of fixation by drift alone, giving 4.4 fixed loci by generation 200 (Fig. 4).

To investigate the importance of population size the simulation model was run for 50 generations over a range of population sizes: the number of loci was kept fixed at 10 and each population size was replicated 10 -
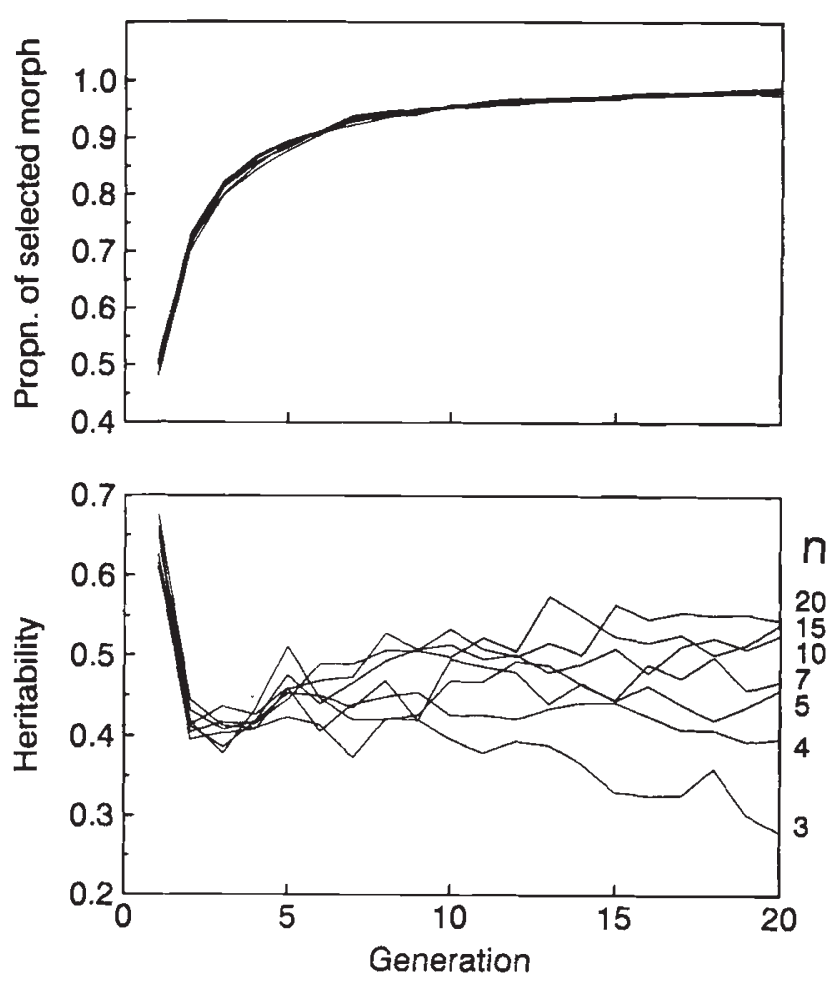

Fig. 2 Simulation results for selection on a dimorphic trait. The underlying trait is determined by $n$ loci each with two alleles that take the value 0 or 1 . The phenotypic value of the underlying trait is equal to the sum of the allelic values plus a normally distributed environmental deviate with mean zero and variance $V_{\mathrm{E}}$. The value on the 0,1 (dimorphic) scale depends on the value of the underlying variable relative to the threshold, here set at $n$ to make the initial proportion 50 per cent for each morph. Each line is the mean of 20 replicates per number of loci $(n)$. 

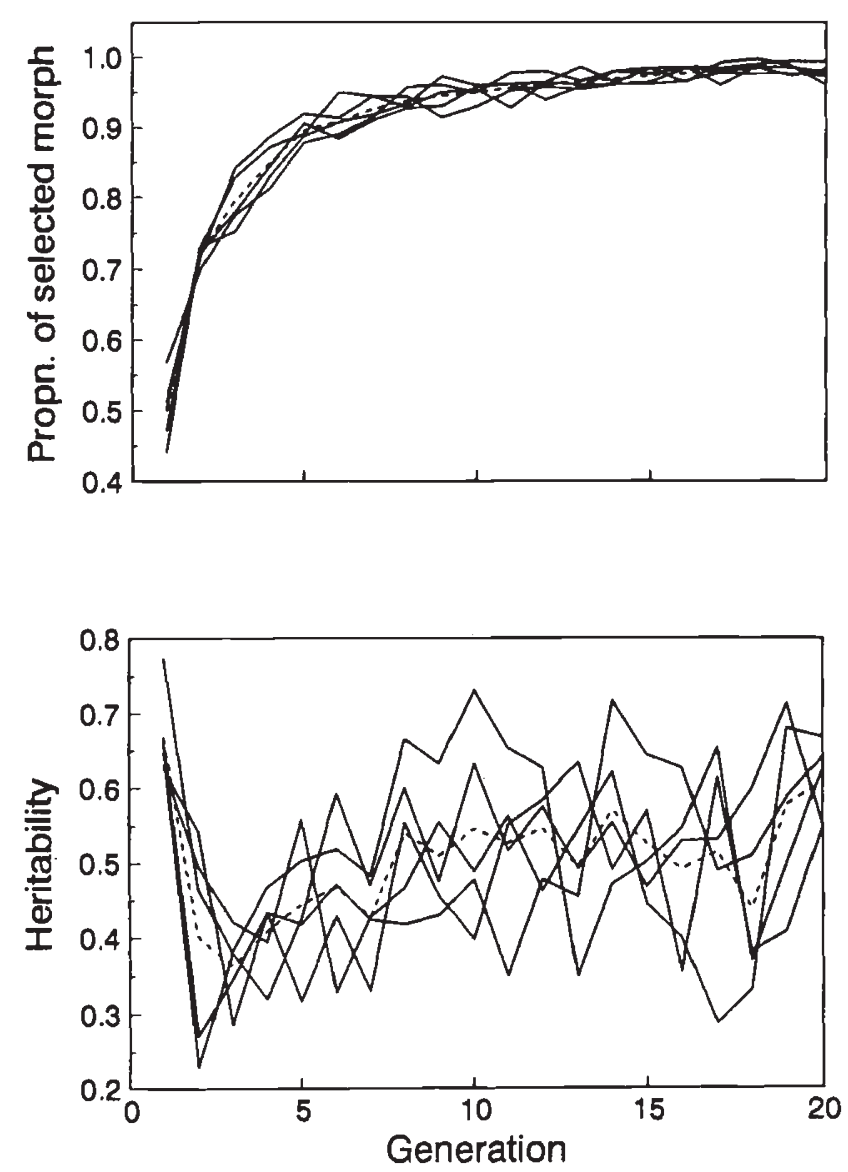

Fig. 3 Five replicates of simulation of selection on a dimorphic trait in which the expression is controlled by the additive effect of 10 loci (see caption to Fig. 2 for further details). (- Individual replicates; $(---)$ mean of the five replicates. fold. At population sizes less than about 50 genetic drift is stronger than selection, the number of loci fixed being very similar with and without selection (Fig. 5). However, the number of loci fixed after 50 generations in the absence of selection decreases rapidly with population size and above approximately 200 less than one locus is fixed by the end of the run (Fig. 5).

\section{Conclusions}

Truncation selection on a dimorphic trait is fundamentally different from truncation selection on a continuously varying trait in that selection intensity in the former case declines over time. As a consequence, selection can change the frequency of a particular morph in a population from 50 per cent to over 95 per cent without the fixation of any alleles (Fig. 2). This result holds even if there are as few as three loci with two alleles per locus determining the trait. The phenotypic trajectory resulting from selection does not depend to any significant degree upon the number of loci coding for the trait but the heritability does (Fig. 2). Simulation results indicate that for a modest number of loci (more than 10) there will be a small decline in additive variance although variability among individual selection lines is likely to be very large (Figs 2 and 3 ).

The important message from the present simulations is that even though directional selection drove the population almost to a monomorphic state (more than 95 per cent of the selected morph) there was comparatively little reduction in genetic variance, or fixation of alleles. Thus, in natural populations the sometimes considerable heritability for the trait (Table 1) may
Fig. 4 Effect of selection and genetic drift on the fixation of loci: selection continued (-), selection discontinued after generation $50(---)$, no selection $(\cdots)$. Each line is the mean of five replicates. Population size $=500$; initial $h^{2}=0.65$; number of loci $=10$.

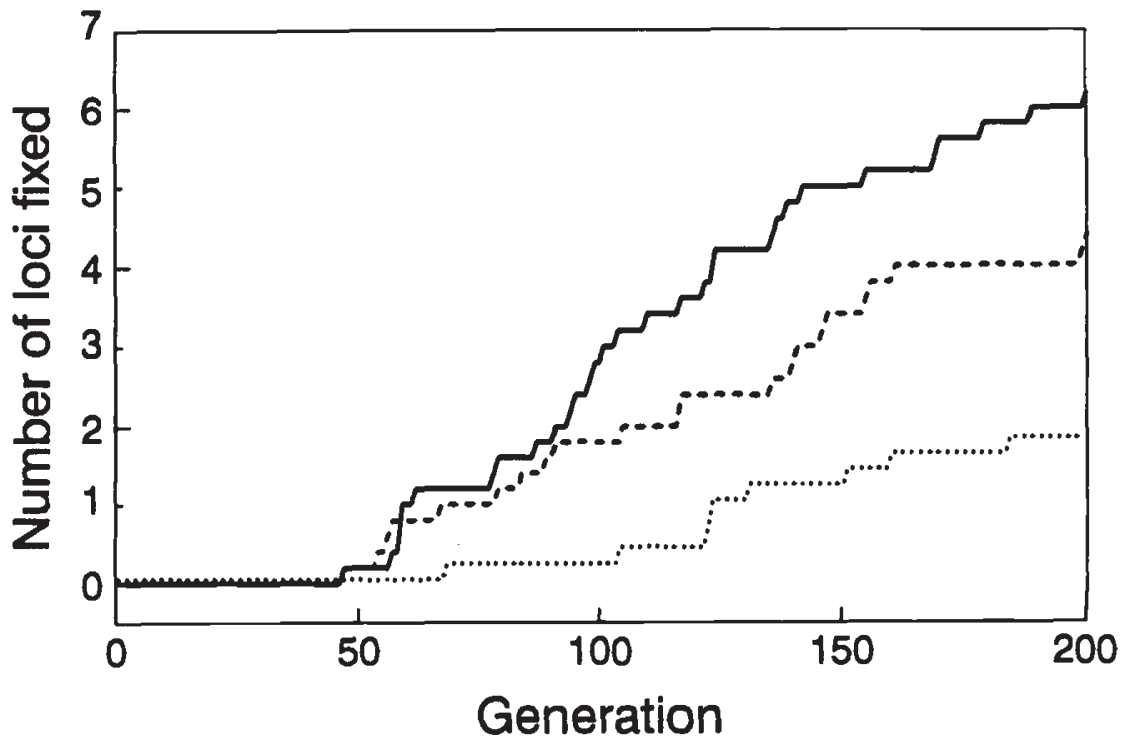




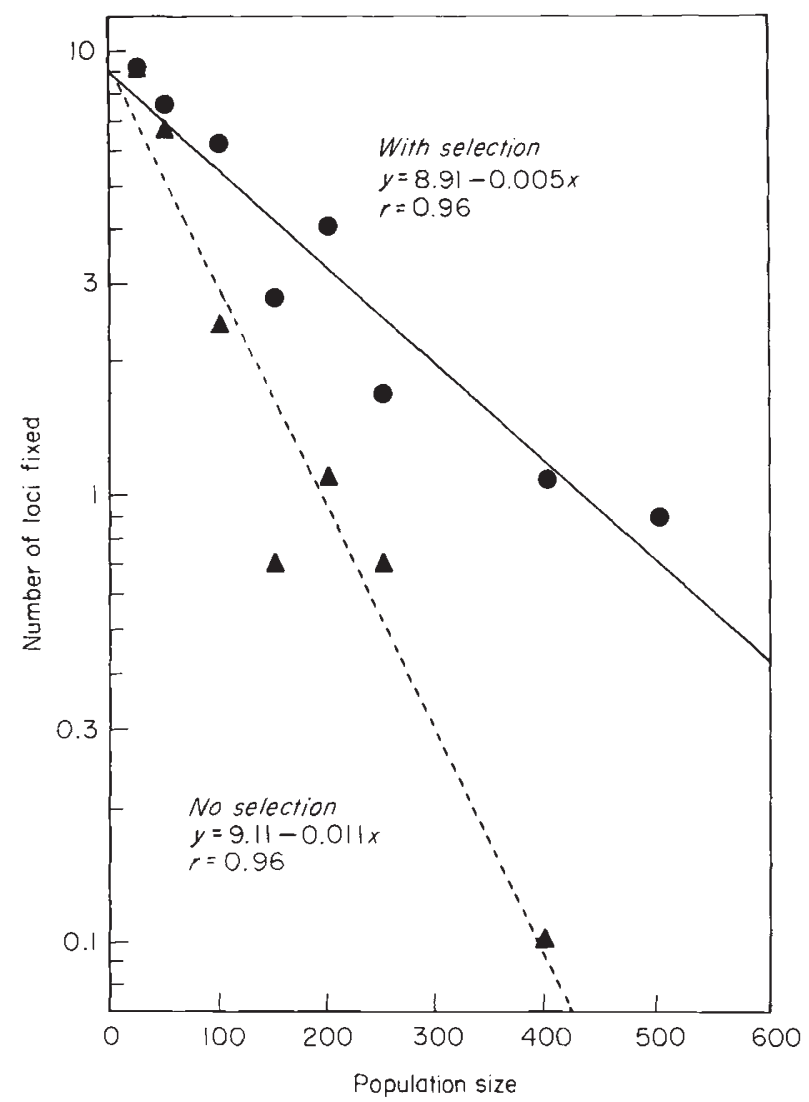

Fig. 5 Number of loci fixed after 50 generations with and without selection for different population sizes. Each point is the mean of 10 replicates. Initial $h^{2}=0.65$; number of loci $=10$.

result in no small measure from the relative inability of strong directional selection to erode genetic variance.

Selection on dimorphic traits is frequently modelled using a game theoretic approach, assuming that selection for a particular morph is frequency-dependent (see, for example, Gross \& Charnov, 1980; Lively, 1986; Hazel et al., 1990; Moran, 1992). While these models have addressed the question of the optimal phenotypic combination they have not addressed the problem of how genetic variation for such traits is maintained. Mani et al. (1990) have shown that frequency-dependent selection on a metrical trait is capable of maintaining considerable genetic variation except when the strength of the frequency-dependence is weak. The present analysis suggests that for dimorphic traits erosion of genetic variance will be very weak even under directional selection: therefore, preservation of genetic variation in threshold traits is likely to be readily obtained even under very weak frequency-dependent selection.

\section{Acknowledgements}

I am most grateful for the helpful comments of Drs D. Fairbairn and Y. Carrière on earlier drafts of this paper. This work was supported by a grant from the Natural Sciences and Engineering Council of Canada.

\section{References}

BULL, J. J., VOGT, R. C. AND BULMER, M. G. 1982. Heritability of sex ratio in turtles with environmental determination. Evolution, 36, 333-341.

BULmer, M. G. 1976. The effect of selection on genetic variability: a simulation study. Genet. Res., 28, 101-117.

BULMER, M. G. 1985. The Mathematical Theory of Quantitative Genetics. Clarendon Press, Oxford, p. 154.

CRESPI, B. J. 1988. Adaptation, compromise and constraint: the development, morphometrics and behavioural basis of a fighter-flier polymorphism in male Hoplothrips karnyi (Insecta: Thysanoptera). Behav. Ecol. Sociobiol., 23, 93-104.

DANFORTH, B. N. 1991. The morphology and behaviour of dimorphic males in Perdita portalis (Hymenoptera: Andrenidae). Behav. Ecol. Sociobiol., 29, 235-247.

DODSON, s. 1989. Predator-induced reaction norms. BioScience, 39, 447-452.

EBERhard, W. G. AND GUTIÉRREZ, E. E. 1991. Male dimorphisms in beetles and earwigs and the question of developmental const raints. Evolution, 45, 18-28.

ELMES, G. W. 1991. Mating strategy and isolation between the two forms, macrogyna and microgyna, of Myrmica ruginodis (Hym. Formicidae). Ecol. Entomol., 16, 411-423.

FAlCONER, D. S. 1989. Introduction to Quantitative Genetics. Longmans, New York.

Gross, M. R. AND CHARNOV, E. L. 1980. Alternative male life histories in bluegill sunfish. Proc. Natl. Acad. Sci. U.S.A., 77, 6937-6940.

HARRIS, R. N., SEMLITSCH, R. D., WILBUR, H. M. AND FAUTH, J. E. 1990. Local variation in the genetic basis of paedomorphosis in the salamander Ambystoma talpoideum. Evolution, 44, 1588-1603.

HAZEL, W. N., SMOCK, R. AND JOHNSON, M. D. 1990. A polygenic model for the evolution and maintenance of conditional strategies. Proc. R. Soc. Lond. B, 242, 181-187.

JANZEN, F. J. 1992. Heritable variation for sex ratio under environmental sex determination in the common snapping turtle (Chelydra serpentina). Genetics, 131, 155-161.

LIVEly, C. M. 1986. Canalization versus developmental conversion in a spatially variable environment. Am. Nature, 128, 561-572.

MACKAY, T. F. C. AND DOYLE, R. W. 1978. An ecological genetic analysis of the settling behaviour of a marine polychaete. 1. Probability of settlement and gregarious behaviour. Heredity, 40, 1-12.

MANI, G. S., CLARKE, B. C. AND SHELTON, P. R. 1990. A model of quantitative traits under frequency-dependent balancing selection. Proc. R. Soc. Lond. B, 240, 15-28. 
MASAKI, S. AND SENO, E. 1990. Effect of selection on wing dimorphism in the ground cricket Dianemobius fascipes (Walker). Bol. San. Veg. Plagas (Fuera de serie), 20, 381-393.

MORAN, N. A. 1992. The evolutionary maintenance of alternative phenotypes. Am. Nature, 139, 971-989.

MORI, K. AND NAKASUJI, F. 1990. Genetic analysis of the wingform determination of the small brown planthopper, Laodelphax striatellus (Hemiptera: Delphacidae). Res. Popul. Ecol., 32, 279-287.

MOUSSEAU, T. A. AND ROFF, D. A. 1989. Adaptation to seasonality in a cricket: patterns of phenotypic and genotypic variation in body size and diapause expression along a cline in season length. Evolution, 43, 1483-1496.

MOUSSEAU, T. A. AND ROFF, D. A. 1989. Geographic variability in the incidence and heritability of wing dimorphism in the striped ground cricket, Allonemobius fasciatus. Heredity, 62, 315-318.
PARKER, E. D. Jr AND ORZACK, S. H. 1985. Genetic variation for the sex ratio in Nasonia vitripennis. Genetics, 110 , 93-105.

ROFF, D. A. 1986a. The evolution of wing dimorphism in insects. Evolution, 40, 1009-1020.

ROFF, D. A. 1986b. The genetic basis of wing dimorphism in the sand cricket, Gryllus firmus and its relevance to the evolution of wing dimorphisms in insects. Heredity, 57, 221-231.

ROFF, D. A. 1990. Selection for changes in the incidence of wing dimorphism in Gryllus firmus. Heredity, 65, 163-168.

ROFF, D. A. 1992. The Evolution of Life Histories: Theory and Analysis. Chapman and Hall, New York.

ROFF, D. A. AND FAIRBAIRN, D. J. 1991. Wing dimorphisms and the evolution of migratory polymorphisms among the insecta. Am. Zool., 31, 243-251. 